\title{
Spongelike alginate nanoparticles as a new potential system for the delivery of antisense oligonucleotides
}

\author{
Aynié I, Vauthier C*, Chacun H., Fattal E., Couvreur P. \\ Université de Paris Sud, UMR 8612, Faculté de Pharmacie, Chatenay-Malabry, France.
}

Manuscript received November 12, 1998, Accepted in revised form February 24, 1999.

Published in: Antisense Nucleic Acid Drug Dev. 1999;9(3):301-12.

doi: 10.1089/oli.1.1999.9.301.

Correspondng author: Christine Vauthier, Université de Paris Sud, UMR CNRS 8612, Faculté de Pharmacie, 5, Rue J.B. Clément, F92296 Chatenay-Malabry Cedex, France.

\begin{abstract}
The aim of this study was to design a new antisense oligonucleotide (ON) carrier system based on alginate nanoparticles and to investigate its ability to protect $\mathrm{ON}$ from degradation in the presence of serum. Pharmacokinetics and tissue distribution of ON-loaded nanoparticles have been determined after intravenous administration. An original and dynamic process for ON loading into polymeric nanoparticles has been applied. It is based on the diffusion of $\mathrm{ON}$ or $\mathrm{ON} /$ polylysine complex into the nanoparticle or the alginate gel, respectively. Indeed, the single coincubation of ON with nanoparticles led, within a few days, to an extremely efficient association. The diffusion kinetic of ON was shown to be dependent on several parameters, incubation temperature, ON concentration, presence or absence of polylysine, polylysine molecular weight, and nanoparticle preparation procedure. This new alginate-based system was found to be able to protect [33P]-radiolabeled ON from degradation in bovine serum medium and to modify their biodistribution, as an important accumulation of radioactivity was observed in the lungs, in the liver, and in the spleen after intravenous administration into mice. ON may be associated efficiently with calcium alginate in a colloidal state. Such nanosponges are promising carriers for specific delivery of ON to lungs, liver, and spleen.
\end{abstract}




\section{Introduction}

Antisense oligonucleotides (ON) are short, single stranded, synthetic sequences of DNA that are complementary to the coding (sense) sequence of intracellular RNA (Rojanasakul, 1996). These fragments can be used to selectively inhibit the synthesis of a define protein. Therefore, they might serve as potential therapeutic agents in cancer therapy and viral infections, blocking the synthesis of the protein responsible for pathologic disorders. Therapeutic applications of antisense ON have been severely hampered by their rapid cleavage by plasma nucleases and their short half-life in the blood. Another limitation to the use of antisense $\mathrm{ON}$ is their ionic character leading to a poor intracellular penetration. One strategy to improve the protection of antisense $\mathrm{ON}$ from degradation and to increase their cell penetration consists in their association with colloidal drug carriers (Chavany et al., 1994, Milhau et al., 1996, Fattal et al., 1998) and poly(alkylcyanoacrylate) nanoparticles display better stability in the presence of nucleases and increased cell penetration (Chavany et al., 1994). In vitro, in some experimental models, the efficiency of antisense ON associated with liposomes or nanoparticles was found to be considerable increased when compared with the free antisense ON (Roper et al., 1993). In vivo, an anti-ras ON administered with the aid of poly(alkylcyanoacrylate) nanoparticles markedly inhibited Ha-ras-dependent tumor growth in nude mice after intratumoral (i.t. injection (Schwab et al., 1994). After i.v. administration, Nakada et al. (1996) showed that antisense $\mathrm{ON}$ associated with poly(alkylcyanoacrylate) nanoparticles presents some limitations because of the mode of interactions of ON with these nanoparticles. ON are attached to the surface of the nanoparticles via ionic interactions with adsorbed quaternary ammonium surfactant molecules (Chavany et al., 1994). This seems to be insufficient to achieve complete protection of ON from nuclease degradation when nanoparticles are administered i.v. This was attributed to the accessibility of the adsorbed $\mathrm{ON}$ to nucleases as well as to a partial desorption of ON from the nanoparticle surface by plasma components (Nakada et al., 1996). In addition, quaternary ammoniums are known to display toxic effects when administered by i.v. Thus, the entrapment of ON into the nanoparticle core instead of being adsorbed onto their surface my be considered a challenge for efficient protection of ON.

In this report, we proposed a new carrier system for the transport of ON based on alginate nanosystems crosslinked with the aid of poly-L-lysine (PLL). It was assumed that the excess of positive lysyl residues (which are not already engaged in ionic bonds with the negatively charged guluronic and mannuronic acids of alginate macromolecules) could interact ionically with ON, leading to their efficient association with the alginate particles. Thus, in this study, ON association has been evaluated and the mechanisms of loading has been investigated in detail. Moreover, the stability of the nanoparticle-associated ON was controlled, as was the ability of those particles to modify tissue distribution after i.v. administration into mice.

\section{Materials and Methods}

Sodium alginate, a natural polysaccharide (61\% mannuronic acid and $39 \%$ guluronic acid) (MW 75000-100000), and five different PLL, hydrobromide (MW: 3900, 7900, 11800, 20500, and 52000), were purchased from Sigma (St. Louis, MO). Calcium chloride was provided by Prolabo (Paris, France). All chemicals were used without further purification. All aqueous solutions were prepared with distilled water that was filtered on $0.22 \mu \mathrm{m}$ filters. 
Phosphodiester antisense ON targeted to the AUC region of the env mRNA of Friend Retrovirus (5'TGAACACGCCATGTC-3') was synthesized by Eugentec (Seraing, Belgium). 5'-radiomabeled ON was obtained from [ $\gamma^{33} \mathrm{P}$ ]ATP (DuPont NEM, Les Ulis, France) (specific activity $74 \mathrm{TBq} / \mathrm{mmol}$ ) using T4 polynucleotide kinase (Biolabs/Ozyme, Montigny-le-Bretonneux, France) and purified according to a classic protocol. The labeled-ON purity was controlled by a method previously described using polyacrylamide gel electrophoresis coupled with a Multi-Channel-Radioactivity Counter (MCRCPAGE) (Aynié et al., 1996. The labelled ON was then diluted with concentrated solutions of nonlabelled ON (final specific activity 0.04-0.4 TBq/mmol).

\section{Preparation of nanoparticles}

Alginate nanoparticle preparation included two steps (Rajaonarivony et al., 1993). First, gelation of a sodium alginate solution $(0.063 \% \mathrm{w} / \mathrm{v}, 950 \mu \mathrm{L})$ was induced by the addition of calcium chloride (18 $\mathrm{mM}, 50 \mu \mathrm{L}$ ) under magnetic stirring to obtain a calcium alginate pregel. Second, PLL was added to form a polyelectrolyte complex with the free remaining negative charges of the pregel (Rajaonarivony et al., 1993), leading to a colloidal system (nanoparticles). Several PLL of different MW (3900, $7900,11800,20500$ and 50200$)$ were used at different concentrations $(0.004 \%$, $0.008 \%$ and $0.016 \% \mathrm{w} / \mathrm{v}$ ) in the final nanoparticle preparation.

Two modes of ON incorporation into these alginate nanoparticles have been investigated. In the first mode, ON were added during alginate nanoparticle formation via the formation of a complex with PLL, as shown in protocol 1 in figure 1. Oligonucleotide-poly-L-lysin complex (ON/PLL complex) was added to calcium alginate pregel under magnetic stirring for $30 \mathrm{~min}$ at ambient temperature. To control ON association with PLL, protocol 1 was followed except that calcium alginate pregel was replaced by the same volume of water. In the second mode, ON was added 4 days after preparation of the unloaded nanoparticles stored at $4^{\circ} \mathrm{C}$ (protocol 2 in figure 1). As previously, the suspension containing $\mathrm{ON}$ was maintained undle gentle magnetic stirring for $30 \mathrm{~min}$ at ambient temperature. In both preparation modes, nanoparticle samples were incubated with IN and stored at $20^{\circ} \mathrm{C}$ for a few days to induce loading.

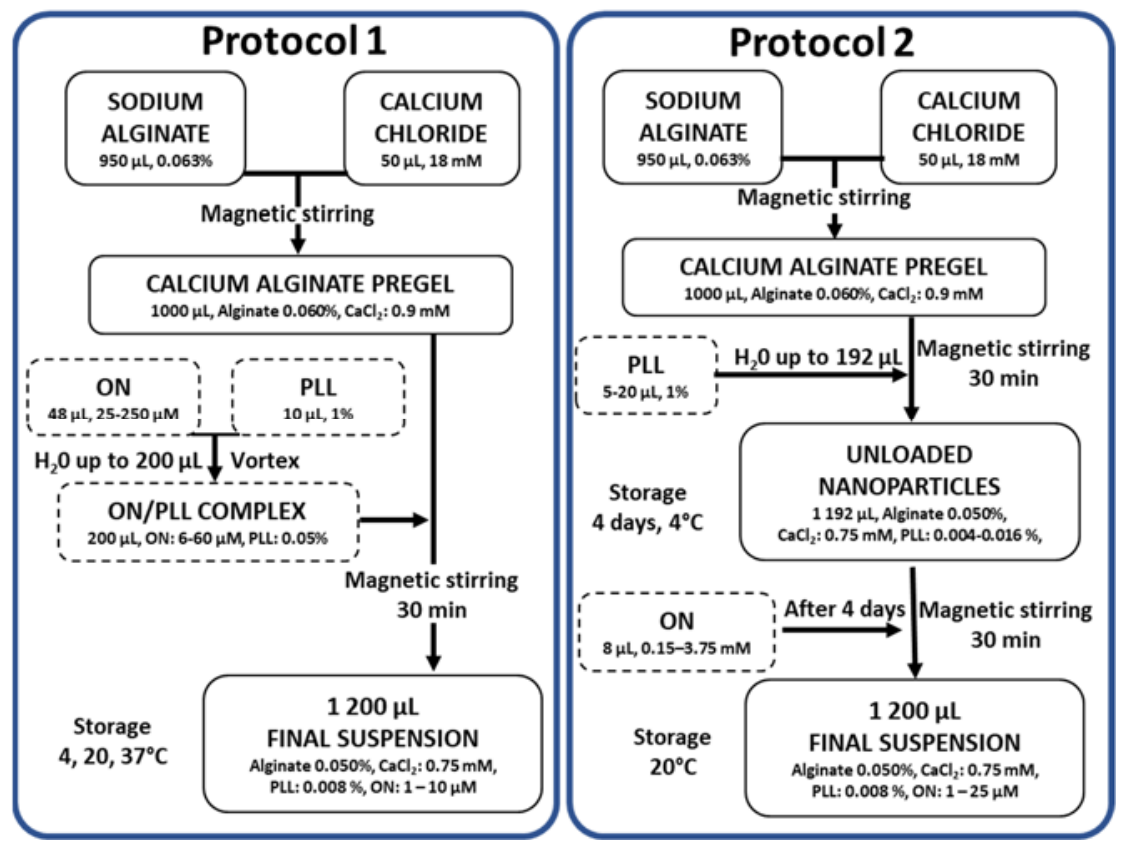

Figure 1: Alginate nanoparticle preparation process: In protocol 1, ON was added as ON/PLL complex to calcium alginate pregel. In protocol 2, ON was added to the nanoparticles already containing PLL. 
The influence of the coincubation temperature was studied with ON-loaded nanoparticles suspensions obtained according to protocol 1. Briefly, a nanoparticle suspension was prepared with an $\mathrm{ON}$ concentration of $1 \mu \mathrm{M}$ at $20^{\circ} \mathrm{C}$. The resulting suspension was divided into three fractions stored at different temperatures $\left(4^{\circ} \mathrm{C}, 20^{\circ} \mathrm{C}\right.$ and $\left.37^{\circ} \mathrm{C}\right)$. Four $\mathrm{ON}$ concentrations, $(1,5,10$, and $25 \mathrm{mM})$ were tested to study the influence of $\mathrm{ON}$ concentration on nanoparticle association efficiency. The charge ratio between anionic phosphate residues of 15-mer IN and cationic lysyl residues of PLL ( $r$ /+) were $0.024,0.12,0.24,0.06$ for $O$ concentrations of $1,5,10$, and $25 \mathrm{mM}$, respectively and PLL concentrations of 10 and $21 \mu \mathrm{M}(0.008 \%)$. The molar ratio of PLL of $3900 \mathrm{MW}$ and ON of MX 4950 $\mathrm{MW}\left(\mathrm{R}_{\mathrm{M} \text {-PL/ON }}\right)$ were $10,2,1$, and 0.4 for $\mathrm{ON}$ concentrations of $1,5,10$, and $25 \mu \mathrm{M}$, respectively and PLL concentration was $10 \mu \mathrm{M}$.

\section{Particle size analysis}

The apparent hydrodynamic diameter of the nanoparticles was determined without suspension dilution by quasielastic light scattering using a Nanosizer ${ }^{\circledR}$ N4 MD (Coultertronics, Margency, France).

\section{Zeta potential measurements}

The zeta potential of the alginate nanoparticles was determined by measuring the electrophoresis mobility using a zeta sizer ${ }^{\circledR}$ (Series 7032 Multi8 Correlator (Malvern, Orsay, France) without further dilution of the suspensions.

Determination of $\mathrm{ON}$ association rate with alginate nanoparticles.

Quantitative determination by MCRC-PAGE analysis. The amount of ON associated with the nanoparticles was investigated at different times ( $3 \mathrm{~h}$ [day 0 on figures 2 and 6 ] and 1, 2, 3, 4, 5, and 6 days after $\mathrm{ON}$ addition to alginate pregel). The association rate was determined using MCRC-PAGE (Aynié et al., 1996). Briefly, electrophoresis was performed on polyacrylamide gels (20\% T [total gel concentration, $\mathrm{g} / 100 \mathrm{~mL}$ ], 3.3\% C [crosslinking agent BIS/total monomers], urea $7 \mathrm{M}, 160 \times 160 \times 1.5$ $\mathrm{mm}$ ) in a lagoon apparatus at $300 \mathrm{~V}$ and $30 \mathrm{~A}$ (generator Consort, Bioblock Scientific, Illkirch, France. The run buffer ( $\mathrm{pH}$ 7.4) contained Tris $90 \mathrm{mM}$, Borate $90 \mathrm{mM}$, and EDTA $2 \mathrm{mM}$. After migration, gels were dried under vacuum (Rapid gel drier, ATTO Corp., Japan) and analyzed using a multichannel radioactivity counter (Automatic TLC-linear Analyzer, Berthold, Wildbad, Germany). In this method, electropherogram profiles showed peaks of radioactivity corresponding to the spots of radiolabeled molecules. Integration of peaks allowed quantitative analysis of the associated radioactivity (Aynié et al., 1996).

All electrophoretic profiles of crude preparations of ON-loaded nanoparticles showed two peaks of radioactivity (Aynié et al., 1996). The first peak appeared at the level of the well, and the second peak was at the same distance of migration as the control ON. The radioactivity of the second peak was measured to evaluate the amount of free ON remaining in the overall alginate nanoparticle preparations. The amount of ON associated with the nanoparticles was deduced from the difference between the initial amount of ON involved in nanoparticle preparation and the amount of ON remaining unassociated as measured by electrophoresis. To control eventual ON desorption from nanoparticles during electrophoretic migration, two extreme voltage conditions (30 and $1000 \mathrm{~V}$ ) were applied. Results concerning $\mathrm{ON}$ association efficiency were compared with those obtained after a run performed at $300 \mathrm{~V}$.

Quantitative analysis by size exclusion chromatography. The ON-loaded nanoparticles were separated from the free ON by Sepharose-4B column $(14 \mathrm{~mL})$ with an appropriate elution buffer (Tris $50 \mathrm{mM}, \mathrm{pH}$ 8.1). Measurement of the radioactivity by liquid scintillation in each collected fraction of $1 \mathrm{~mL}$ allowed plotting of a chromatogram. Peaks were identified by passage through a column of dextran blue (void volume $6 \mathrm{~mL}$ ), pure $\mathrm{ON}$ (elution volume $12 \mathrm{~mL}$ ), and pure ON/PLL complex (elution volume $12 \mathrm{~mL}$ ) used as controls. 
In vitro stability of nanoparticle-associated $\mathrm{ON}$ in bovine serum.

Free $\mathrm{ON}$ or nanoparticle-associated $\mathrm{ON}(50 \mu \mathrm{L})$ was added to $166 \mu \mathrm{L}$ of non-decomplemented fetal bovine serum (FSB). Samples were incubated for $0,2,5,10,30$, and $60 \mathrm{~min}$ at $37^{\circ} \mathrm{C}$. Immediately after incubation at $37^{\circ} \mathrm{C}, 20 \mu \mathrm{M}$ of a $20 \% \mathrm{NaOH}$ solution was added to the samples, which were heated to $70^{\circ} \mathrm{C}$ for $15 \mathrm{~min}$ in order to block serum enzymatic activity. Finally, $25 \mathrm{mg}$ sodium citrate was dissolved in the samples to pump the calcium out of the alginate calcium gel and to promote the release of ON from the nanoparticles. The stability of the ON was evaluated by MCRC-PAGE after 18 $h$ of incubation in sodium citrate at ambient temperature. Samples of free ON and nanoparticleassociated $\mathrm{ON}$ were incubated at $37^{\circ} \mathrm{C}$ in water for $60 \mathrm{~min}$, and the same post incubation procedure was applied to check that these conditions were not destructive for the ON.

\section{Animal experiments}

OF1 mice ( 5 weeks, $20 \mathrm{~g}$ ) received in the tail vein $200 \mu \mathrm{L}$ of free ON or ON nanoparticles (prepared according to protocol 1) at a dose of $10 \mathrm{nmol} / 10 \mathrm{~mL} / 3 \mathrm{MBq} / \mathrm{kg}$. This dosage corresponded to a dose of alginate polymer of $5 \mathrm{mg} / \mathrm{kg}$. For pharmacokinetics studies, animals were euthanized $5,15,30,60$, 120 and $240 \mathrm{~min}$ and $24 \mathrm{~h}$ after i.v. administration. Organ distribution studies were conducted at 5, 15, 30, 60, 120 and $240 \mathrm{~min}$ and $24 \mathrm{~h}$ after administration. A control consisting of ON/PLL complex was also injected, and mice were euthanized 5 min after the injection. For all tested animals, blood, and organs (liver, lungs, spleen, bone marrow, and kidneys) were removed. Plasma recovered from blood and organs were dissolved using $1 \mathrm{~mL}$ of Soluen ${ }^{\circledast} 35^{\circ}$ (Packard) before determination of radioactivity by scintillation counting. Results were expressed as pmol ON/g organ for biodistribution studies and as $\mathrm{fmol} / \mu \mathrm{M}$ plasma for pharmacokinetic studies.

\section{Results}

\section{Oligonucleotide unloaded alginate nanoparticle preparation.}

Prior to studying $\mathrm{ON}$ association with alginate nanoparticles, preparation conditions for ON-unloaded nanoparticles were investigated. Five PLL of different MW (3 900, 7900,11 800, 20 500, 50 200) and three final PLL concentrations expressed as weight/volume percentage $(0.004 \%, 0.008 \%$ and $0.016 \%$ $\mathrm{w} / \mathrm{v}$ ) were used. Table 1 gives the size and zeta potential of the obtained nanoparticles. For a PLL concentration in the final nanoparticle suspension of $0.008 \%$, nanoparticle size was found to increase depending on the PLL MW.

Table I: Size and zeta potential of ON-loaded alginate nanoparticles $(n=3-10)$.

\begin{tabular}{|c|c|c|c|c|}
\hline Mw of PLL & $\begin{array}{c}\text { Lysyl residue number } \\
\text { (average per PLL chain) }\end{array}$ & $\begin{array}{c}\text { Final PLL } \\
\text { concentration } \\
(\% \mathrm{w} / \mathrm{v})\end{array}$ & $\begin{array}{c}\text { Nanoparticle size } \\
(\mathrm{nm})\end{array}$ & $\begin{array}{c}\text { Nanoparticle zeta } \\
\text { potential } \\
(\mathrm{mV})\end{array}$ \\
\hline \multirow{2}{*}{3900} & \multirow{2}{*}{30} & 0.004 & $210 \pm 30$ & ND* $^{*}$ \\
\cline { 3 - 5 } & \multirow{2}{*}{7400} & 0.008 & $268 \pm 33$ & $-37.6 \pm 1.0$ \\
\hline 11800 & 92 & 0.004 & $278 \pm 43$ & $-47.5 \pm 0.8$ \\
\hline 20500 & 160 & 0.008 & $305 \pm 43$ & $-38.0 \pm 1.9$ \\
\hline 50200 & 392 & 0.016 & $310 \pm 50$ & $-31.4 \pm 0.5$ \\
\hline & & 0.008 & $389 \pm 67$ & $-36.8 \pm 2.5$ \\
\hline
\end{tabular}

*ND: not determined 
Nevertheless, zeta potential was not significantly modified $(-38 \mathrm{mV})$ by PLL chain length for similar concentrations used $(0.008 \%)$. In fact, nanoparticle size remained constant (around $300 \mathrm{~nm}$ ) whatever the concentration of $7900 \mathrm{MW}$ PLL used $(0.004 \%, 0.008 \%, 0.016 \% \mathrm{w} / \mathrm{v})$, whereas the higher the concentration of PLL, the lower was the negative value of the zeta potential.

\section{Association of ON/PLL ionic complex with alginate pregel (protocol 1)}

For ON association with alginate nanoparticles, PLL of $7900 \mathrm{MW}$ at a concentration of $0.008 \% \mathrm{w} / \mathrm{v}$ $(10 \mu \mathrm{M})$ was chosen. Some loaded nanoparticle samples were also prepared with PLL of $3900 \mathrm{MW}$ at $0.008 \% \mathrm{w} / \mathrm{v}(21 \mu \mathrm{M})$ in order to compare ON association efficiency as a function of the PLL chain length. In protocol 1, ON/PLL was added as an ionic complex to the pregel.

Influence of time of incubation on ON loading. The amount of ON associated as ON/PLL ionic complex with the alginate pregel is shown in figure 2. Determination by MCRC-PAGE analysis were performed at different time intervals on nanoparticle preparations containing 1, 5, $10 \mu \mathrm{M}$ ON and stored at $20^{\circ} \mathrm{C}$. The resulting association of $\mathrm{ON}$ with alginate nanoparticles appeared time dependent.

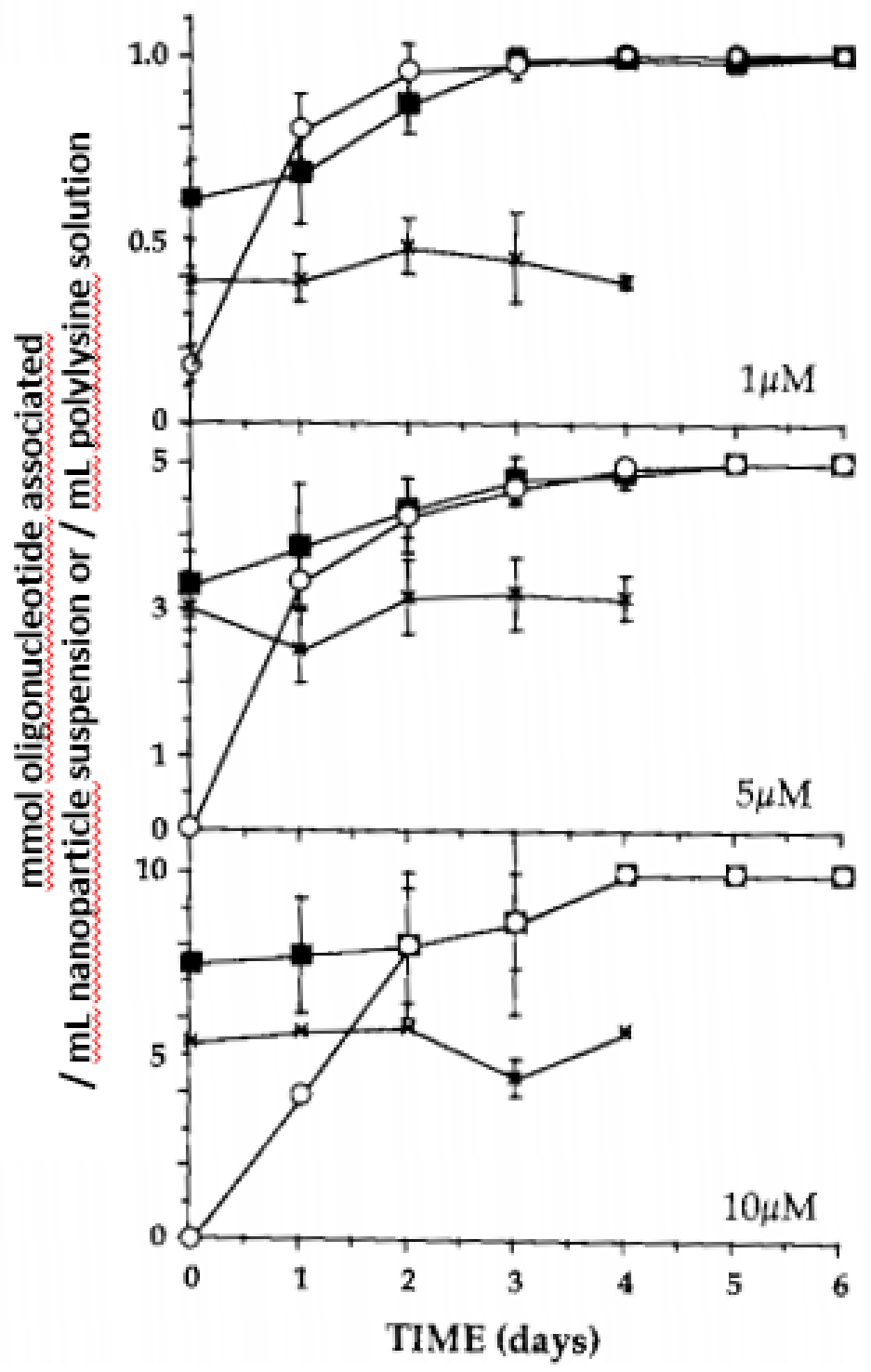

Figure 2: Association kinetics of ON/PLL complex with alginate pregel (protocol 1) (closed squares), of ON with PLL alone (crosses), or of ON alone with alginate pregel (open circles). ON concentrations: 1 , 5 , and $10 \mu \mathrm{M}$. 
After 4 days, association yields of $1,4,10 \mathrm{nmol} \mathrm{ON}$ associated $/ \mathrm{mL}$ nanoparticle suspension (corresponding to 2, 10 and, $20 \mathrm{nmol} \mathrm{ON} / \mathrm{mg}$ alginate) were obtained for respective ON concentrations of $1,5,10 \mu \mathrm{M}$, which corresponded to an ON-loading efficiency of $100 \%$. Thus, the amount of $\mathrm{ON}$ that associated with alginate nanoparticles increased proportionally with the ON concentration in the incubation medium, and no saturation could be observed at least up to $10 \mu \mathrm{M}$. As a control, the addition of ON to PLL in a single aqueous solution was studied under the same conditions. In the presence of PLL alone, around $50 \%$ of the ON were found associated with the PLL as a complex right after sample preparation and at whatever the ON concentration was. The level of association remained constant over the period of investigation. Consequently, ON association was more important with alginate than with PLL alone at any sampling time. A second control consisted of the single addition of ON to the calcium alginate pregel but without PLL (Figure 2). A kinetic of association could be obtained, but the initial association was found to be closed to zero. Therefore, ON association with the pregel was far less rapid in the absence of PLL than with PLL. The molecular weight of PLL (3 900 or 7900 ) had no influence on the ON association kinetics for the range of ON concentration tested (1-10 $\mu \mathrm{M})$ (data not shown). Whatever the ON concentration was, the zeta potential and size of the obtained nanoparticles remained unchanged during the association kinetics, displaying the same values as the unloaded nanoparticles (respectively - $34 \mathrm{mV}$ ad $320 \mathrm{~nm}$ ) (data not shown).

As shown in figure 3, whatever the applied voltage for electrophoretic runs $(30,300$, and $1000 \mathrm{~V})$, the ON association with PLL alone (complex), and with nanoparticles (ON/PLL added to the pregel) remained unchanged at around $50 \%$ for the ON/PLL complex and $100 \%$ for nanoparticles. Thus, the electric field did not modify interactions between $\mathrm{ON}$ and PLL, and between $\mathrm{ON}$ and nanoparticles.

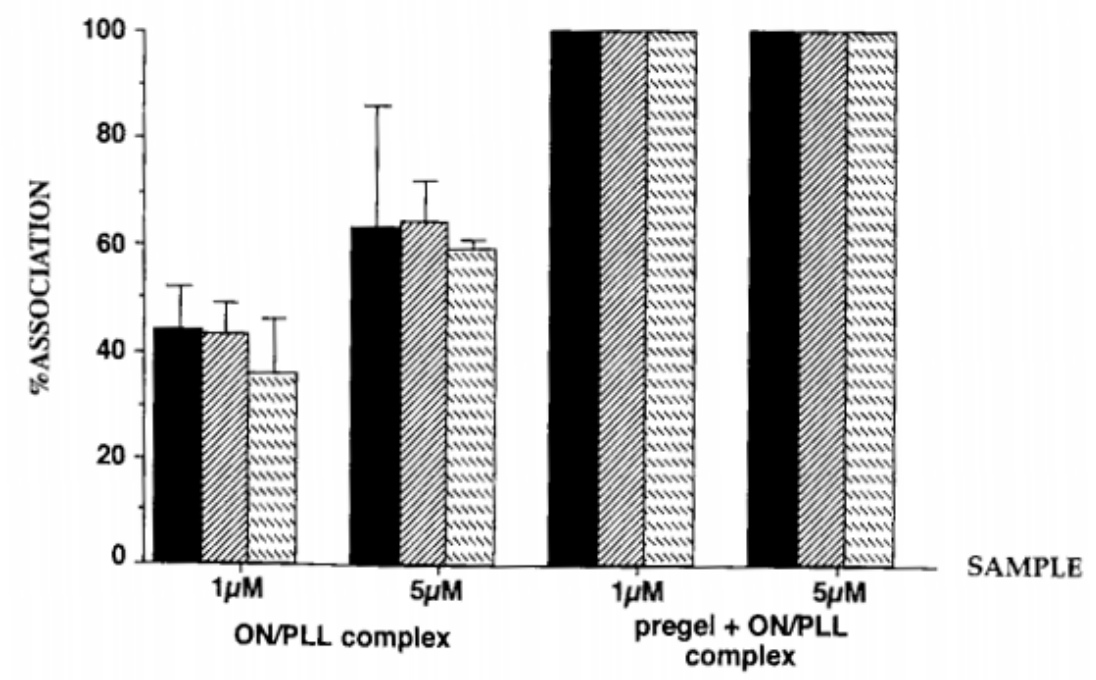

Figure 3: Influence of voltage on the association of ON with PLL alone (at equilibrium) or of ON/PLL complex with the pregel (4 days of incubation). Voltage: $30 \mathrm{~V}$ (black), $300 \mathrm{~V}$ (diagonal stripes), $1000 \mathrm{~V}$ (doted lines).

The association efficiency of ON with nanoparticles was also investigated through an exclusion chromatograph assay for $\mathrm{ON}$ concentration conditions of $1 \mu \mathrm{M}$. The resulted chromatograms (Figure 4) showed that the ON-loaded nanoparticle sample gave a single peak at an elution volume of $6 \mathrm{~mL}$ that was distinct for the ON/PLL complex (elution volume $12 \mathrm{~mL}$ ) and ON control (elution volume 12 $\mathrm{mL}$ ). This clearly confirmed that $\mathrm{ON}$ molecules were completely associated with alginate 3 days after nanoparticle preparation. 


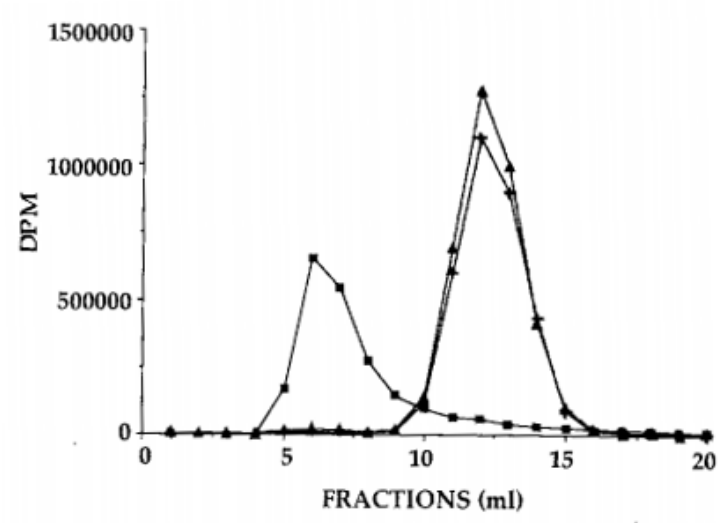

Figure 4: Quantitative analysis by size exclusion chromatography (at $1 \mu \mathrm{M}$ ON concentration): (squares): ON-loaded nanoparticles 3 days after preparation (protocol 1); (crosses) ON/PLL complex alone; (triangles) free $\mathrm{ON}$ as controls.

Influence of the temperature of loading. The effect of temperature on the rate of $\mathrm{ON}$ association with alginate nanoparticles was investigated over 4 days. As shown in the figure 5 , the association of ON with alginate nanoparticles appeared to be slightly temperature dependent. The increase in the incubation temperature led to a faster association process. At $4^{\circ} \mathrm{C}$, the association of $\mathrm{ON}$ with alginate nanoparticles was lowest and remained constant over the duration of the experiment $(70 \%)$. At $37^{\circ} \mathrm{C}$, the rate of association was still faster than at $20^{\circ} \mathrm{C}$, and $100 \%$ association was reached after 2 days of incubation at $37^{\circ} \mathrm{C}$, whereas it required 3 days at $20^{\circ} \mathrm{C}$.

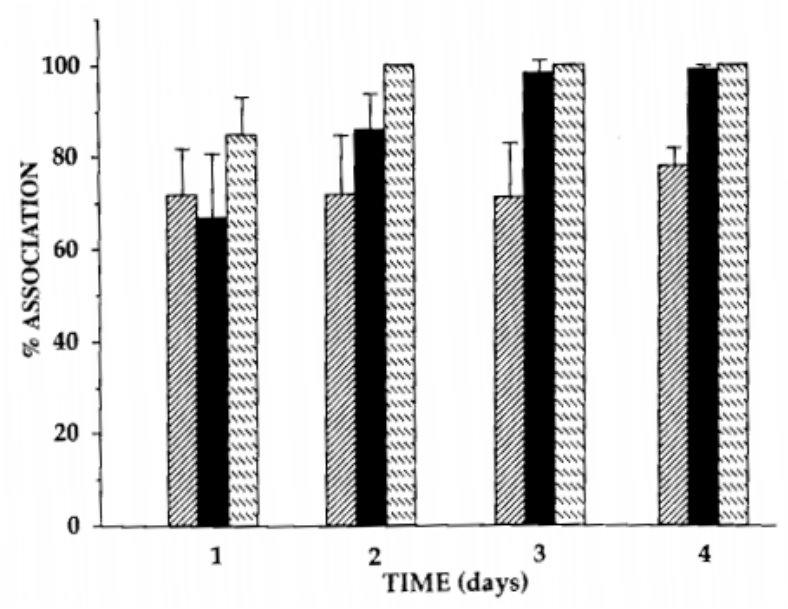

Figure 5: Effect of temperature on ON association with alginate nanoparticles (protocol 1). ON-loaded nanoparticles were prepared at $1 \mu \mathrm{M}$ ON concentration at $20^{\circ} \mathrm{C}$ and then stored at $+4^{\circ} \mathrm{C}$ (diagonal stripes), $20^{\circ} \mathrm{C}$ (black) and $37^{\circ} \mathrm{C}$ (doted lines).

\section{Association of ON with prepared alginate nanoparticles (protocol 2).}

ON association with alginate nanoparticles resulting from protocol 2 (addition of ON to alginate nanoparticles already containing PLL) revealed a time-dependent association whatever the ON concentration used $(1,5,10$, and $25 \mu \mathrm{M}$ ) and the PLL molecular weight (3 900 or 7900 ) (figure 6). All ON molecules observed to be associated with nanoparticles whatever the concentration of ON used. The higher the concentration of ON, the faster was the rate of association. However, the time needed to achieve total association of ON increased with the concentration of ON because the increase in speed was less important than the increase in concentration (figure 6). Data from figure 2 
were plotted in figure 6 to allow a clear comparison with the loading process using the addition of the ON/PLL complex to alginate pregel (protocol 1). Such a comparison led to the observation that the addition of ON to alginate nanoparticles already containing PLL (protocol 2) induced a lower initial (at day 0 ) association rate than later addition of ON/PLL complex to the alginate pregel (protocol 1). However, the $100 \%$ ON nanoparticle association was reached more rapidly using protocol 2 than with protocol 1, and loading was more rapid tant with the single addition of ON to the pregel without PLL (figure 6). In contrast, with protocol 1, the initial association was highest when PLL with a higher MW was used.

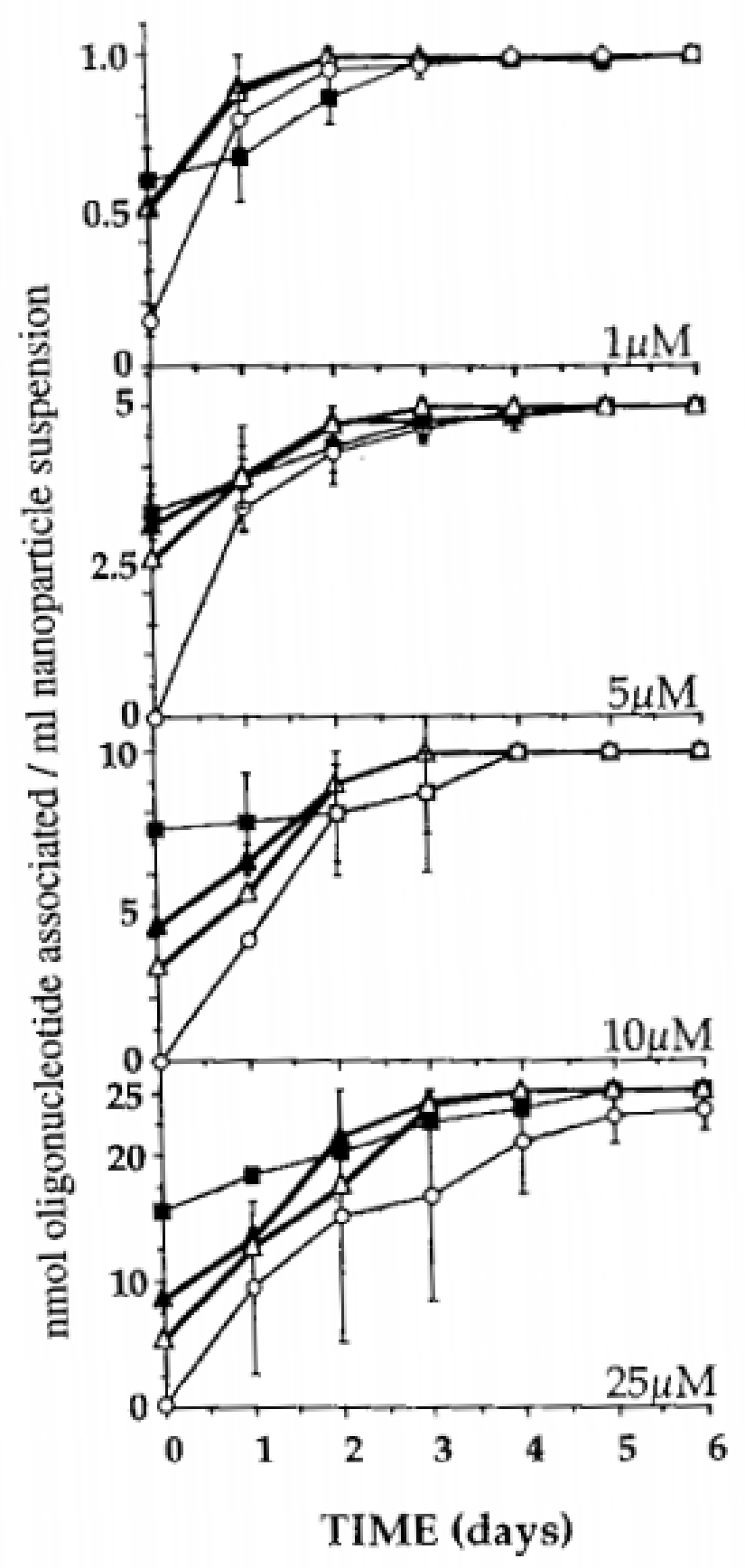

Figure 6: Association kinetics of ON with alginate nanoparticles containing PLL at $3900 \mathrm{MW}$ (open triangles) or $7900 \mathrm{MW}$ (closed triangles) (protocol 2) with increasing ON concentrations (1, 5, 10 and $25 \mu \mathrm{M})$. Addition of ON/PLL complex to the pregel (squares) and of ON alone to the pregel (cercles) used as controls. 


\section{In vitro protection of $\mathrm{ON}$ incorporated into alginate nanoparticles.}

The results reported in figure 7 show the percentage of non-degraded ON found after incubation in serum for various incubation times. The curve corresponding to the free ON showed a typical degradation profile, with a half-life in serum of $6.5 \mathrm{~min}$. In contrast, the ON associated with nanoparticles were highly resistant to degradation by the serum enzymes. After 1 hour of incubation in serum, more than $70 \%$ of the ON remained undegraded, being protected from the action of serum nucleases by the nanoparticles.

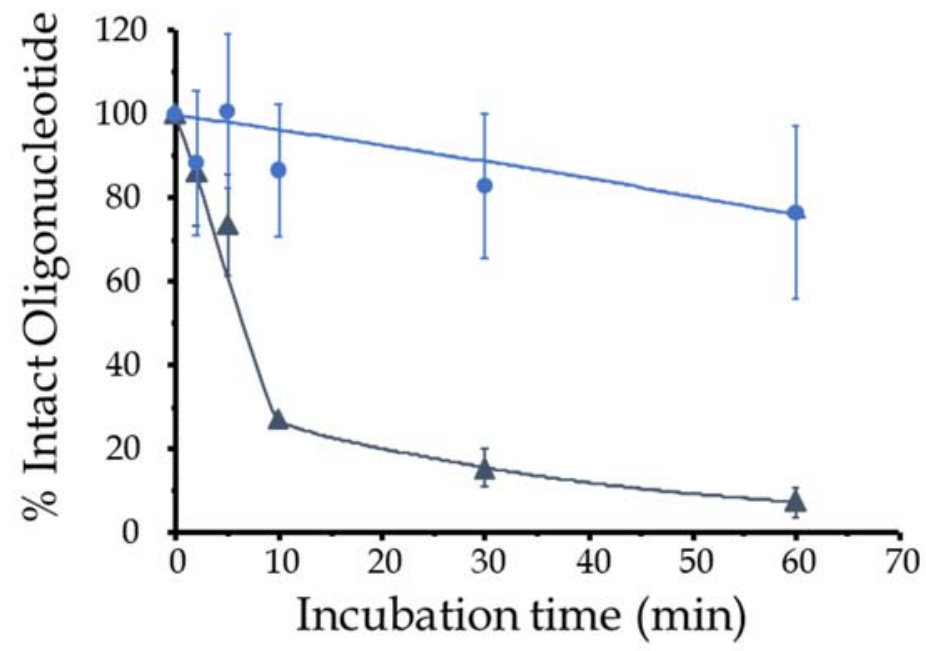

Figure 7: In vitro stability of ON incubated in PBS. Free ON (triangles), ON associated with alginate nanoparticles (circles).

\section{Pharmacokinetics and biodistribution of ON-loaded nanoparticles.}

Radiolabelled ON-loaded nanoparticles were injected into mice to provide organ distribution and plasma pharmacokinetic data. Radiolabelled ON alone and radiolabelled ON/PLL complex (only at 5 $\mathrm{min}$ ) were injected as controls. Plasma concentration profiles (figure 8A) were very similar for both formulations and were characterized by a very short half-life in the distribution phase. This rapid clearance could be attributed to a fast tissue uptake. Tissue disposition profiles for both formulations (Figure 8B,C) showed dramatic differences in the distribution of the free $\mathrm{ON}$ and nanaoparticleassociated ON. Massive acculumation has been observed into the lungs $5 \mathrm{~min}$ after injection of ONloaded nanoparticles and up to $15 \mathrm{~min}$ (around $100 \mathrm{pmol} / \mathrm{g}$ organ). The level of radioactivity then decreased progressively in that organ. With ON-loaded nanoparticles, radioactivity accumulated also in the organs of the mononuclear phagocyte system (MPS), with a maximal concentration $\left(C_{\max }\right)$ at 15 min for the liver ( $88 \mathrm{pmol} O \mathrm{~N} / \mathrm{g}$ organ) and a $\mathrm{C}_{\max }$ at $20 \mathrm{~min}$ for the spleen (100 pmol/g organ). After $30 \mathrm{~min}$ of residence into the liver and $60 \mathrm{~min}$ in the spleen, the radioactivity decreased. Nevetheless, no significant differences were observed between the two formulations in distribution into the kidneys. For the bone marrow, the level of radioactivity was lower with the ON nanoparticles at 15 and $30 \mathrm{~min}$. After $60 \mathrm{~min}$, however, it increased dramatically, reaching a value close to that obtained with free ON. ON/PLL complex behave the same as free ON 5 min after i.v. administration (data not shown). 


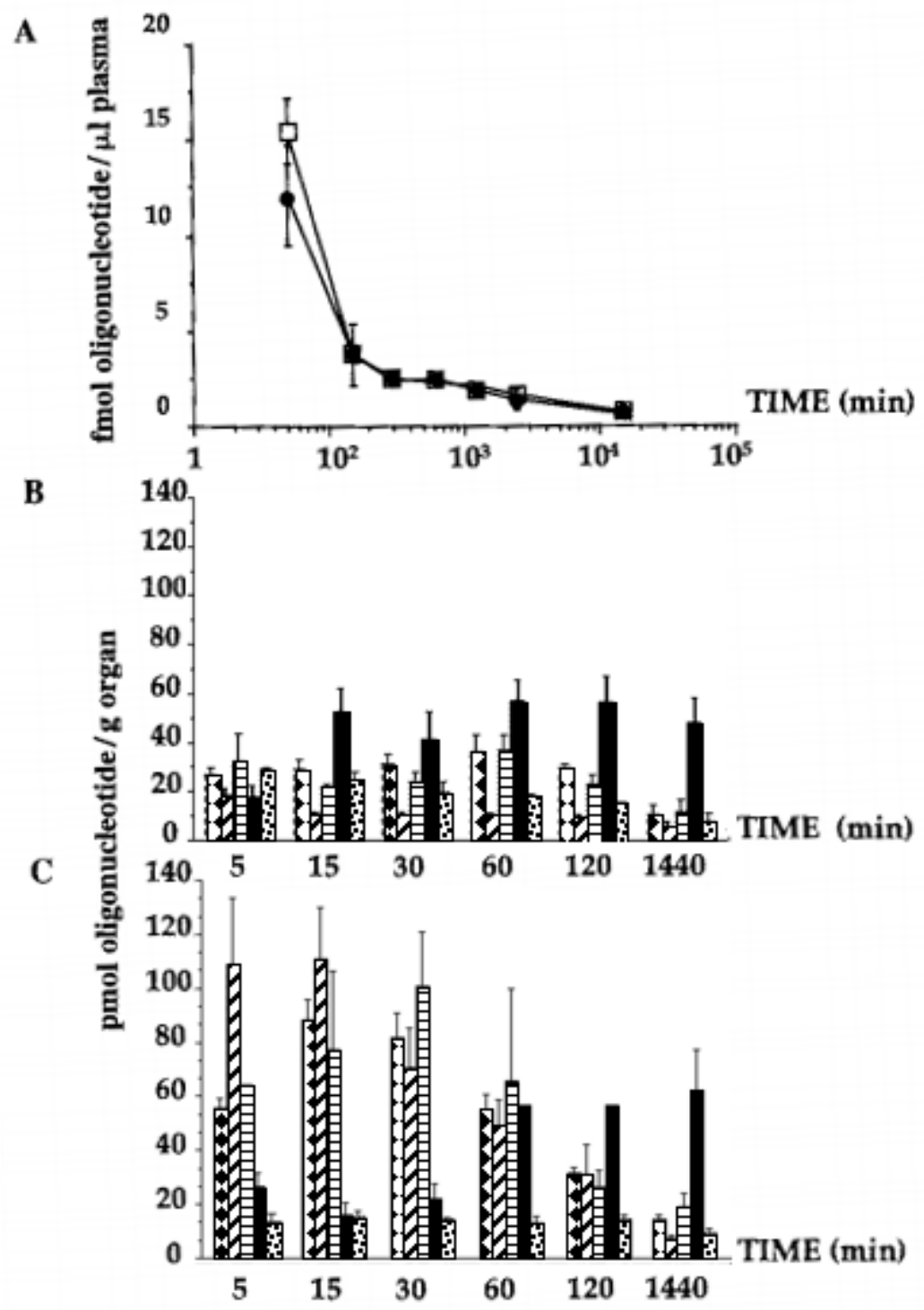

Figure 8: Pharmacokinetics (A) and biodistribution of $O N$ free $(B)$ and $O N$-loaded alginate

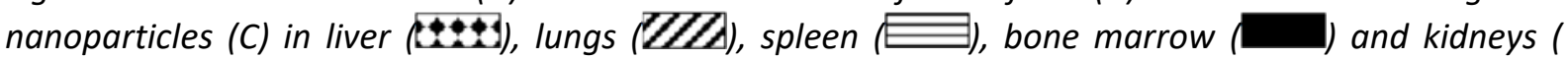
F) up to 24 hours after i.v. into mice. Bars represent $S D(n=5$ for time 5 minutes and $n=3$ for other times).

\section{Discussion}

This study demonstrates that the association of ON with alginate nanoparticles could occur through the single addition of ON/PLL complex to calcium alginate pregel (protocol 1). ON/PLL complexes could be formed by ionic interactions involving amino groups of PLL and phosphate groups of the ON (Citro et al., 1992). ON/PLL complex formation took place almost instantaneously, and an equilibrium was reached corresponding to the coexistence of free PLL and ON with ON-bound PLL.

When preparing ON-loaded nanoparticles, a characteristic Tyndall effect appeared immediately after the addition of the ON/PLL complex to the calcium alginate pregel. Furthermore, according to light scattering measurements, the addition of ON/PLL complex instead of PLL alone to calcium alginate pregel did not affect nanoparticle formation. This indicated that nanoparticle formation occurred even if part of the PLL was initially complexed with the ON. 
Comparing results of the association of ON with the nanoparticles, and the association of ON with PLL, it can be observed that the amount of ON binding the nanoparticle was higher than the amount that associated with PLL alone. This clearly suggests that at least a part of the ON molecules was able to interact directly with the calcium alginate pregel. This is confirmed by the fact that ON were able to associate with the calcium alginate pregel even in the absence of PLL. The nature of the interactions occurring between $\mathrm{ON}$ and calcium alginate pregel is not clearly understood, but it may be hypothesized that calcium ions could form a bridge between carboxylic acids of uronic residues of alginate polymer and phosphate groups of ON. Some authors have described strong interactions between calcium and phosphate residues of nucleic acids (Marynick and Schaefer, 1975, Duguid et al., 1995). Moreover, it is well known that calcium ions bind to alginate polymer, and preferentially to the polyguluronic acids to give an egg-box structure (Morris et al., 1978).

It is noteworthy that the presence of PLL allows improvement in the kinetics of ON/nanoparticle interaction. This may be explained by the fact that PLL plays the role of an efficient shuttle for the ON to diffuse rapidly into the gel (step 1 of protocol 1 ). This diffusional process for ON leading is relevant to the observation that the surface potential of the particles was not modified after complete loading of ON, which strongly suggested that the $\mathrm{ON}$ were incorporated inside the nanoparticle matrix rather than simply adsorbed onto their surface (step 2 of protocol 1). Indeed, it could have been expected that the surface accumulation of polyanions, such as ON, onto alginate nanoparticles would have induced a significant zeta potential modification (Fattal et al., 1998).

ON incorporation into the gel could be achieved by a diffusion mechanism involving constant rearrangements of the ionic interactions occurring between alginate and PLL and between PLL and $\mathrm{ON}$. The existence of such a diffusion mechanism is also supported by the effect of the temperature on the rate of $\mathrm{ON}$ association with alginate nanoparticles. Indeed, an increase in temperature in the gel should promote chain mobility and tend to decrease, at the same time, the intensity of the interactions between molecules (De Gennes, 1982). The combination of these effects should facilitate the diffusion of macromolecules, such as $\mathrm{ON}$, inside the nanoparticles, leading to the faster rate of incorporation observed at $37^{\circ} \mathrm{C}$ (figure 5).

It was also possible to efficiently associate $\mathrm{ON}$ with nanoparticles by simple addition of the $\mathrm{ON}$ to the already formed nanoparticles (protocol 2). However, the initial association was lower, as PLL was already associated with alginate and consequently less accessible to ON than in the protocol 1 (where PLL was entirely available). Moreover, the initial concentration of ON was highest with PLL of $7900 \mathrm{MW}$. This is understandable, as increasing the PLL chain length promotes the probability of having interactions with $\mathrm{ON}$ and alginate on the same chain. On the other hand, ON loading with protocol 2 was more rapid than the single addition of ON to the pregel without PLL.

This observation is still relevant, with the initial binding of ON to the remaining free lysyl residues of PLL, which should more efficiently attract the ON through ionic driving forces (step 1 of the protocol 2). Nevertheless, PLL should remain partly localized on the surface of the particles after ON addition, as the zeta potential was unchanged. This also suggests that $\mathrm{ON}$ could move by reptation from the PLL sites on the particle surface to the available positive charges brought by calcium $\left(\mathrm{Ca}^{2+}\right)$ or PLL or both within the core of the gel. The diffusion kinetics could relate to complex conformational rearrangements of macromolecules, as in the second protocol, the diffusion of ON was more rapid than in the first protocol. In fact, it is supposed that the conformational rearrangements of PLL and alginate macromolecules already occurred when protocol 2 was performed.

The suggested mechanism of ON loading using protocols 1 and 2 is summarized in figure 9 . Thus, the system used for the transport of ON may be considered as being a spongelike system of $300 \mathrm{~nm}$ size (nanosponge). 


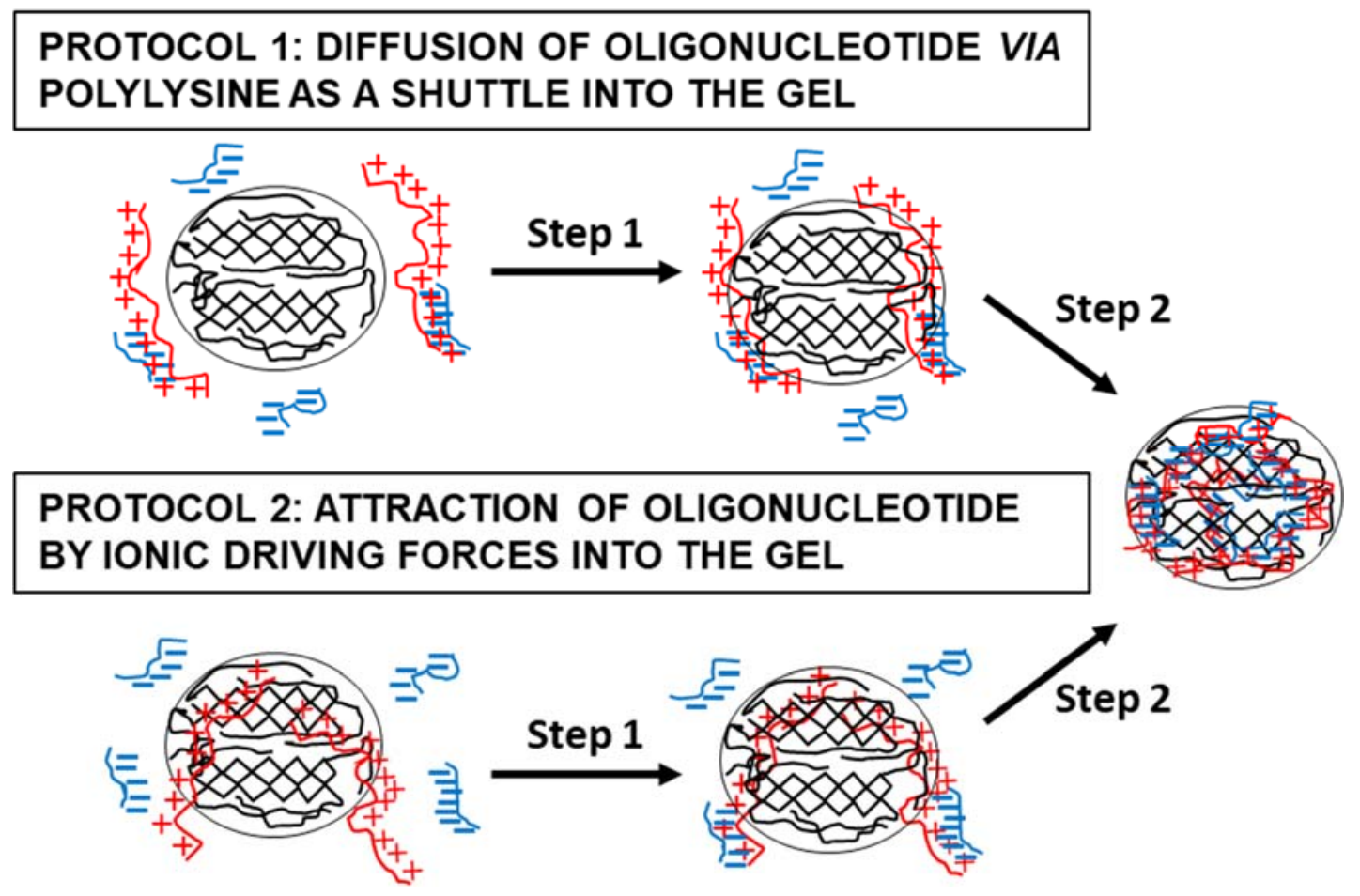

Figure 9: Scheme of ON loading into alginate-nanosponge (protocol 1 and protocol 2). Pregel including alginate chains complexed with calcium ions (Black), oligonucleotide bearing negative charges (Blue), polylysine chains bearing positive charges (red).

From the point of view of pharmaceutical technology this new system has the following advantages. (1) The nanosponges are prepared in an aqueous medium without any organic solvent, which are generally used in all the nanotechnologies available today. (2) The ON loading procedure is very easy. It is based on a single addition of ON/PLL complex to a gel of alginate or a single addition of ON to alginate nanosponges. (3) The ON was found intact in the conditions of preparation of the nanosponges. (4) The $\mathrm{ON}$ are localized in the core of the particles, contrary to the available nanotechnologies, which generally have association of the $\mathrm{ON}$ at the surface of a particle (Chavany et al., 1994, Godard et al., 1995, Fritz et al., 1997, Zobel et al., 1997).

As preliminary in vivo evaluation, we have injected i.v. into mice these alginate nanosponges loaded with radiolabeled $\left[{ }^{33} \mathrm{P}\right]-\mathrm{ON}$ prepared according to protocol 1 . This formulation was found to strongly modify the profile of tissue distribution of the radioactivity compared with a solution of free ON or the complex of IN with PLL. It is noteworthy that PLL alone was unable to modify ON tissue distribution in vivo, as tissue levels were the same for free and complexed ON (ON/PLL complex). This could be because of complete dissociation of the ionic ON/PLL complex in a physiologic medium or displacement of the equilibrium after dilution of the sample in plasma.

The modified tissue distribution of ON associated with alginate nanosponges favored the liver and the spleen, but still more important was the lung capture $(1000 \mathrm{pmol} / \mathrm{g}$ organ at $5 \mathrm{~min}$ after injection). This confirms, as shown after incubation in vitro with serum, that ON remained associated with alginate in vivo without rapid displacement by blood proteins or ions. This is explained by the strong interaction between the $\mathrm{ON}$ and the alginate nanoparticles, which can be mediated by calcium or by PLL. We can speculate that the stability of such an interaction could result from a cooperative effect of ionic interactions involving several consecutive charges placed along the polyelectrolyte chains and interacting. This can cause an increase in the binding strength between $\mathrm{ON}$ and its carrier. 
Capture by the liver and spleen may be explained by the opsonization of the nanosponges, as is widely observed with other poly(ethylene glycol) (PEG)-uncoated colloidal carriers (Illum et al., 1982, Kreuter, 1983, Nakada et al., 1996), although the uptake seems to be less rapid than with other types of nanoparticles (e.g., poly(alkylcyanoacrylate) (Nakada et al., 1996). The observed delay could be due to the more rapid accumulation of nanosponge-associated $\mathrm{ON}$ in the lungs. It is remarkable that lung accumulation was almost 10 -folds greater with alginate nanosponges than with poly(isobutylcyanoacrylate) nanoparticles after i.v. injection of the same amount of polymer (Nakada et al., 1996).

This massive accumulation in the lungs of ON-loaded alginate nanosponges remains unexplained, but there are two possible explanations. First, it may be hypothesized that microemboli were formed in the lungs as a result of aggregation of the nanosponges just after injection. The uptake of larger particles or aggregates $(7-15 \mu \mathrm{m})$ by the lung can be explained by their entrapment in the pulmonary capillary bed before being distributed to the whole body. The smaller particles $(0.3-3$ $\mu \mathrm{m}$ ), however, are phagocytized by the liver after passing the pulmonary filter (Illum et al., 1982, Kreuter, 198"). In fact, aggregation of injected nanoparticles is generally observed after fast $(1 \mathrm{~mL} / 5 \mathrm{sec})$ but not after slow injection $(1 \mathrm{~mL} / \mathrm{min})$ of suspensions (Kreuter et al., 1979). For this reason, the injection rate in these experiments was kept at a slow rate of about $1 \mathrm{~mL} / \mathrm{min}$. Another reason for aggregation could have been a size increase in the nanoparticles through coating with serum and complement components. However, no tendency to particle aggregation in plasma could be detected by size measurement. Finally, in the literature, when embolus-like formations were observed 5 to $15 \mathrm{~min}$ after injection, $16 \%$ of the injected dose of polystyrene nanoparticles (Adlersberg et al., 1969) and $80 \%$ of the injected dose of ON-complexed liposomes (Litzinger et al., 1996) were distributed in the lungs. After disintegration of emboli, these accumulated particulate drug carriers gradually redistributed to liver at, respectively, $4 \mathrm{~h}$ and $18 \mathrm{~h}$ after injection, where approximately $3 \%$ of the injected dose was present in lungs and $85 \%$ was present in the liver in the two cases. In our case, no inverse pharmacokinetic relation of lungs and liver accumulation was obtained (figure 8). Moreover, the ON level in lungs measured at 5 min after injection was only $5 \%$ when expressed as a percentage of the injected dose. For all those reasons, microembolization is unlikely.

Another explanation for the lung retention of nanosponges is the occurrence of specific interactions of the particles with the macrophages present in pulmonary capillaries, as the lung endothelium represents the first portion of the MPS that particles encounter after i.v. injection. Takada et al., (1984) showed that liposomes coated with polysaccharides (amylopectin derivatives) accumulated 35 folds more in lungs than did conventional liposomes. No aggregation of liposomes was detected by electron microscopy. These authors found that a fluorescent probe encapsulated within such liposomes was substantially located in alveolar macrophages and blood monocytes after i.v. injection. Poste et al. (1982) showed that liposomes in the lung capillaries are engulfed by circulating blood phagocytes that subsequently migrate to the alveoli to become alveolar macrophages, as the capillary endothelium is continuous. Moreover, Gonsho et al. (1994) found that the tissue distribution properties of the glycosyl carriers depended on the type of saccharide modified on the carrier and suggested that macrophages probably have specific receptors to recognize each glycosyl carrier. Thus, we speculated that the contribution of alveolar macrophages may be a plausible explanation for the relatively high uptake of ON-loaded alginate nanosponges by the lungs, as alginate is a polysaccharide.

This preliminary in vivo evaluation of alginate nanoparticles has clearly shown that the model ON used was not readily dissociated from the nanoparticles in serum and was efficiently protected from cleavage by serum nucleases. In addition, the particles appeared physically stable over time. These 
properties are important criteria for therapeutic applications. The nanoparticles used in this study preferentially concentrated the ON within the lungs before distributed the ON toward the MPS organs. In agreement with numerous works preformed with PEG-coated colloidal drug carriers with a prolonged residence time within the bloodstream, studies are in progress in our laboratory to use the alginate nanoparticles as a template for the design of a long-term carrier.

In conclusion, the data obtained in this study demonstrate that $\mathrm{ON}$ may be associated successfully with calcium alginate in a colloidal state. These nanosponges may be loaded with ON through a single diffusional mechanism that is accelerated by the use of PLL. This new system was found to protect ON from degradation in PBS medium and to modify the tissue distribution of radiolabeled ON in vivo. It may be a promising carrier for antisense ON delivery.

\section{Acknowledgments}

This work has been partly supported by grant CNRS (GDR Vectola, programme Physique-Chimie du Vivant) and ARC (grant 1188). I.A. was given a followship from the French Ministry of Research (Ph.D.) and was a recipient of a fellowship from ARC.

\section{References}

Adlersberg L, Singer JM, Ende E. Redistribution and elimination of intravenously injected latex particles in mice. J Reticuloendothel Soc. 1969 Oct-Dec;6(5):536-60.

Aynié I, Vauthier C, Foulquier M, Malvy C, Fattal E, Couvreur P. Development of a quantitative polyacrylamide gel electrophoresis analysis using a multichannel radioactivity counter for the evaluation of oligonucleotide-bound drug carrier. Anal Biochem. 1996 Sep 5;240(2):202-9. doi: 10.1006/abio.1996.0350.

Chavany C, Saison-Behmoaras T, Le Doan T, Puisieux F, Couvreur P, Hélène C. Adsorption of oligonucleotides onto polyisohexylcyanoacrylate nanoparticles protects them against nucleases and increases their cellular uptake. Pharm Res. 1994 Sep;11(9):1370-8. doi: 10.1023/a:1018923301967.

Citro G, Perrotti D, Cucco C, D'Agnano I, Sacchi A, Zupi G, Calabretta B. Inhibition of leukemia cell proliferation by receptor-mediated uptake of c-myb antisense oligodeoxynucleotides. Proc Natl Acad Sci U S A. 1992 Aug 1;89(15):7031-5. doi: 10.1073/pnas.89.15.7031

De Gennes P.G. The Formation of Polymer/Polymer Junctions. In: Microscopic aspects of adhesion and lubrification. J.M. Georges Ed. Elsevier Scientific Publishing Company, Amsterdam. Tribology Series 1981; 7: 355-367. DOI: 10.1016/S0167-8922(08)70894-0.

Duguid J, Bloomfield VA, Benevides J, Thomas GJ Jr. Raman spectroscopy of DNA-metal complexes. I. Interactions and conformational effects of the divalent cations: $\mathrm{Mg}, \mathrm{Ca}, \mathrm{Sr}, \mathrm{Ba}, \mathrm{Mn}, \mathrm{Co}, \mathrm{Ni}, \mathrm{Cu}, \mathrm{Pd}$, and $\mathrm{Cd}$. Biophys J. 1993 Nov;65(5):1916-28. doi: 10.1016/S0006-3495(93)81263-3. PMID: 8298021; PMCID: PMC1225927.

Fattal E, Vauthier C, Aynie I, Nakada Y, Lambert G, Malvy C, Couvreur P. Biodegradable polyalkylcyanoacrylate nanoparticles for the delivery of oligonucleotides. J Control Release. 1998 Apr 30;53(1-3):137-43. doi: 10.1016/s0168-3659(97)00246-0.

Fritz H, Maier M, Bayer E. Cationic Polystyrene Nanoparticles: Preparation and Characterization of a Model Drug Carrier System for Antisense Oligonucleotides. J Colloid Interface Sci. 1997 Nov 15;195(2):272-88. doi: 10.1006/jcis.1997.5172.

Godard G, Boutorine AS, Saison-Behmoaras E, Hélène C. Antisense effects of cholesterol-oligodeoxynucleotide conjugates associated with poly(alkylcyanoacrylate) nanoparticles. Eur J Biochem. 1995 Sep 1;232(2):40410.

Illum L, Davis SS, Wilson CG, Thomas NW, Frier M, Hardy JG. Blood clearance and organ deposition of intravenously administered colloidal particles. The effects of particle size, nature and shape. Int J Pharm 1982; 12:135-146. DOI: 10.1016/0378-5173(82)90113-2 
Kreuter J. Evaluation of nanoparticles as drug-delivery systems. II: Comparison of the body distribution of nanoparticles with the body distribution of microspheres (diameter greater than 1 micron), liposomes, and emulsions. Pharm Acta Helv. 1983;58(8):217-26.

KreuterJ, Täuber U, Illi V. Distribution and elimination of poly(methyl-2-14C-methacrylate) nanoparticle radioactivity after injection in rats and mice. J. Pharm Sci 1979; 68: 1443-1447. Doi: 10.1002/jps.2600681129.

Marynick DS, Schaefer HF 3rd. Theoretical studies of metal-phosphate interactions: interaction of $\mathrm{Li}+, \mathrm{Na}+, \mathrm{K}+$, $\mathrm{Be}++, \mathrm{Mg}++$, and $\mathrm{Ca}++$ with $\mathrm{H} 2 \mathrm{PO} 4-$ and $(\mathrm{CH} 3 \mathrm{O}) 2 \mathrm{PO} 2-:$ implications for nucleic acid solvation. Proc Natl Acad Sci U S A. 1975 Oct;72(10):3794-8. doi: 10.1073/pnas.72.10.3794.

Milhaud PG, Bongartz JP, Leubleu B, Philippot JR. pH-Sensitive Liposomes and Antisense Oligonucleotide Delivery. Drug Delivery 1996; 3(2):67-73. Doi: 10.3109/10717549609031175.

Morris ER, Rees DA, Thom D. Boyd J. Chiroptical and stoichiometric evidence of a specific, primary dimerisation process in alginate gelation. Carbohydrate Res 1978; 66(1): 145-154. Doi: 10.1016/S0008-6215(00)83247-4.

Nakada Y, Fattal E, Foulquier M, Couvreur P. Pharmacokinetics and biodistribution of oligonucleotide adsorbed onto poly(isobutylcyanoacrylate) nanoparticles after intravenous administration in mice. Pharm Res. 1996 Jan;13(1):38-43. doi: 10.1023/a:1016017014573.

Poste G, Bucana C, Raz A, Bugelski P, Kirsh R, Fidler IJ. Analysis of the fate of systemically administered liposomes and implications for their use in drug delivery. Cancer Res. 1982 Apr;42(4):1412-22.

Rajaonarivony M, Vauthier C, Couarraze G, Puisieux F, Couvreur P. Development of a new drug carrier made from alginate. J Pharm Sci. 1993 Sep;82(9):912-7. doi: 10.1002/jps.2600820909.

Rojanasakul Y. Antisense oligonucleotide therapeutics: drug delivery and targeting. Adv Drug Deliv Rev 1996; 18(2):115-131. Doi: 10.1016/0169-409X(95)00020-8.

Ropert C, Malvy C, Couvreur P. Inhibition of the Friend retrovirus by antisense oligonucleotides encapsulated in liposomes: mechanism of action. Pharm Res. 1993 Oct;10(10):1427-33. doi: 10.1023/a:1018910922633.

Schwab G, Chavany C, Duroux I, Goubin G, Lebeau J, Hélène C, Saison-Behmoaras T. Antisense oligonucleotides adsorbed to polyalkylcyanoacrylate nanoparticles specifically inhibit mutated Ha-ras-mediated cell proliferation and tumorigenicity in nude mice. Proc Natl Acad Sci U S A. 1994 Oct 25;91(22):10460-4. doi: 10.1073/pnas.91.22.10460.

Takada M, Yuzuriha T, Katayama K, Iwamoto K, Sunamoto J. Increased lung uptake of liposomes coated with polysaccharides. Biochim Biophys Acta. 1984 Nov 28;802(2):237-44. doi: 10.1016/0304-4165(84)90167-3.

Zobel HP, Kreuter J, Werner D, Noe CR, Kümel G, Zimmer A. Cationic polyhexylcyanoacrylate nanoparticles as carriers for antisense oligonucleotides. Antisense Nucleic Acid Drug Dev. 1997 Oct;7(5):483-93. doi: 10.1089/oli.1.1997.7.483. 